\title{
EDITORIAL \\ Anterior cervical fusion and rhBMP-2: a prospective study is needed to assess optimal dosing and delivery
}

\author{
Thomas J. Buell, MD, and Christopher I. Shaffrey, MD \\ Department of Orthopaedic and Neurological Surgery, Duke University, Durham, North Carolina
}

$\mathrm{S}$ OME prior reports have suggested that the use of recombinant human bone morphogenetic protein-2 (rhBMP-2) in anterior cervical fusion (ACF) may be beneficial in select patients with risk factors for developing pseudarthrosis. ${ }^{1}$ However, other reports have provided evidence to suggest that there is potential for high complication rates (e.g., hematoma/seroma, cervical swelling, dysphagia) when using rhBMP-2 for ACF. ${ }^{2}$ As such, there are currently no clear guidelines or definitive recommendations regarding the usage of rhBMP-2 in patients undergoing ACF. The explanation for some of the discrepancies from prior rhBMP-2/ACF reports is likely multifactorial but could potentially be attributed to nonstandardized dosing and delivery of rhBMP-2. ${ }^{1-4}$ To address this important topic, Mendenhall et al. performed a retrospective study to evaluate the safety and efficacy of low-dose rhBMP-2 for use in patients undergoing ACF (both anterior cervical discectomy and corpectomy). ${ }^{5}$ The authors hypothesized that the higher doses of rhBMP-2 used in prior ACF studies may have led to the higher complication rates observed.

In this study, Mendenhall and colleagues included a total of 198 patients (62 [31\%] were smokers) who underwent stand-alone ACF (median of 2 levels fused; mean rhBMP-2 dose $0.50 \pm 0.09 \mathrm{mg} /$ level; both contained and noncontained delivery devices were used). The authors reported associated complications and fusion rates with a minimum of 1 year of radiographic follow-up. Briefly, $22(11 \%)$ patients experienced dysphagia, $11(6 \%)$ patients experienced cervical swelling, 2 (1\%) patients underwent washout for hematoma, $1(0.5 \%)$ patient had washout for seroma, $2(1 \%)$ patients had pseudarthrosis requiring posterior cervical fusion, and $3(2 \%)$ patients experienced a new postoperative neurological deficit (which was reported to have recovered by the last follow-up). Regarding rates of arthrodesis, study results demonstrated that 190 (96\%) patients had solid arthrodesis over an average of 15 months of follow-up (with no statistically significant difference in fusion rates between smokers and nonsmokers). After carefully analyzing these study results, the authors concluded that use of low-dose rhBMP-2 may safely and effectively augment ACF and could potentially benefit select patients at risk for pseudarthrosis (e.g., smokers, multilevel ACF).

The current study provides novel data that may impact surgical decision-making and could assist preoperative patient counseling. To better understand the potential impact of this study, it may be beneficial to briefly review the early safety and efficacy studies of rhBMP-2 usage for spine surgery. The first clinical studies of rhBMP-2 for spinal arthrodesis were performed in patients with lumbar degenerative disease ${ }^{6,7}$ In 2000, Boden et al. reported their results from the first human clinical pilot trial assessing the feasibility of rhBMP-2 in single-level lumbar degenerative disease. ${ }^{6}$ In their study, patients received a tapered cylindrical threaded fusion cage filled with either rhBMP-2/collagen sponge or autogenous iliac crest bone (control). ${ }^{6}$ The study results demonstrated that arthrodesis occurred more reliably in those patients treated with rhBMP-2-filled fusion cages compared with controls. ${ }^{6}$ The authors reported no adverse events related to the rhBMP-2 treatment. ${ }^{6}$

Following this initial clinical pilot trial, Burkus et al. reported results from a multicenter prospective 2-year study comprised of 279 patients with degenerative lumbar disc disease. 7 In this follow-up study, patients underwent lumbar interbody fusion using two tapered threaded fusion cages, and the investigational group received rhBMP-2 on an absorbable collagen sponge. The study results demonstrated that lumbar fusion using rhBMP-2 and a tapered titanium fusion cage yielded solid union and could potentially eliminate the need for harvesting iliac crest bone graft. ${ }^{7}$ These positive results culminated in FDA approval being granted to Medtronic for the use of rhBMP-2 in patients undergoing anterior lumbar interbody fusion (ALIF).

Since then, there has been increasing use of rhBMP-2 in an off-label fashion, especially in patients with known risk factors for pseudarthrosis. This was demonstrated in an 
exploratory meta-analysis reported by Hofstetter et al. in 2016. ${ }^{4}$ The authors reported the dose-dependent effectiveness and morbidity of rhBMP-2 after assessing its use in a variety of common spinal arthrodesis procedures, which included anterior cervical discectomy and fusion (ACDF), posterior cervical fusion, ALIF (the only FDA-approved indication), transforaminal lumbar interbody fusion, posterior lumbar interbody fusion, and posterolateral lumbar fusion. ${ }^{4}$ Other authors have also reviewed the literature and reported similar findings, highlighting the increasing trend of off-label rhBMP-2 usage for spinal arthrodesis. ${ }^{8-10}$

When off-label rhBMP-2 is used in the anterior cervical spine, some authors have hypothesized that the putative inflammatory effect (which contributes to the effectiveness of rhBMP-2 for arthrodesis) may spread to adjacent cervical structures and lead to increased postoperative morbidity (e.g., hematoma/seroma, cervical swelling, dysphagia). ${ }^{2}$ Notably, a study by Shields et al. demonstrated high complication rates in patients undergoing ACF with high-dose rhBMP-2 $(2.1 \mathrm{mg} / \mathrm{level}){ }^{2}$ The authors reported that 23\% (35/151) of patients experienced complications including hematoma/seroma $(\mathrm{n}=15)$ and prolonged hospital stay or readmission because of swallowing/breathing issues or severe swelling without hematoma $(n=13)$.

However, more recent literature has demonstrated some evidence of safety in usage of lower rhBMP-2 doses and especially when these were delivered in contained devices. ${ }^{1,11}$ Tumialán et al. reviewed 200 patients who underwent a single- or multilevel ACDF with PEEK spacers filled with a collagen sponge impregnated with low-dose rhBMP-2. In their report, the authors concluded that the incidence of symptomatic dysphagia may be reduced with lower doses of rhBMP-2 that are placed only within the PEEK spacer. ${ }^{1}$ Despite these positive results, due to the potential high complication rates previously reported (where various doses were being utilized with different delivery devices), the FDA issued a public health notification in 2008 detailing the risks of rhBMP-2 in the anterior cervical spine. ${ }^{12}$

The current study by Mendenhall et al. revisits this important topic and provides novel evidence to suggest safety and efficacy of low-dose rhBMP-2 in ACF. ${ }^{5}$ Compared with previous studies that utilized a variety of dosing regimens ranging from 0.6 to $2.1 \mathrm{mg}$ per level fused, the current authors utilized a lower average dose of rhBMP-2 $(0.50 \pm 0.09 \mathrm{mg} / \mathrm{level})$. A potential weakness of the study was that both contained and noncontained delivery devices were utilized. This is an important consideration given that a recent article by Maza et al. suggested that a contained delivery route could significantly decrease postoperative cervical swelling complications. ${ }^{11}$ However, the authors addressed this limitation in their discussion and reported no difference in dysphagia or cervical swelling when comparing delivery systems. Notably, the authors reported that complication rates (dysphagia, cervical swelling, hematoma/seroma) were similar to the rates reported for ACF without rhBMP-2 usage. ${ }^{5}$

Another important consideration for patients undergoing ACF using rhBMP-2 is perioperative steroid administration. Some recent studies have suggested that prophylactic administration of steroids could reduce postoperative cervical swelling complications. ${ }^{11,13}$ Maza et al. reported that postoperative tapered steroids (oral and intravenous) significantly decreased respiratory compromise following ACF using low-dose rhBMP-2. ${ }^{11}$ Also, in a separate study, Liu et al. reported that perioperative intravenous and local steroid use (locally administered depomedrol on a collagen sponge) could reduce incidence and severity of early dysphagia after ACF using rhBMP-2..$^{13}$ Despite these positive results, in the current study by Mendenhall et al., prophylactic steroids were not administered (note that only patients with symptoms related to cervical swelling received postoperative steroids). ${ }^{5}$ As such, this is a topic (along with contained vs noncontained delivery) that likely needs further investigation when low-dose rhBMP-2 is used for ACF.

In conclusion, we commend the authors of the current study. ${ }^{5}$ Mendenhall et al. have provided novel data that may suggest safety and efficacy using low-dose rhBMP-2 in patients undergoing ACF. ${ }^{5}$ However, in addition to dosing of rhBMP-2, other important considerations include the delivery system and perioperative steroid administration-whether an oral, intravenous, or local application at the time of surgery. In the context of previous articles that investigated ACF and off-label rhBMP-2 usage, these current results reemphasize and highlight the need for a more definitive multicenter prospective evaluation of the topic. A prospective investigation could help determine the optimal dose and delivery of rhBMP-2 (as well as perioperative steroid protocol) for promoting cervical arthrodesis while minimizing associated complications. Considering the FDA-generated warning letter from 2008, any potential postoperative complications related to off-label usage of rhBMP-2 in ACF may have medicolegal risks and negative implications. As with any medical procedure, balancing the risks and benefits should be carefully considered during preoperative planning and thoroughly discussed with patients. As such, a prospective study of ACF and rhBMP-2 that rigorously assesses standardized dosing strategies, delivery systems/interbody cages, and perioperative steroid protocols may provide significant benefits to both surgeons and patients.

https://thejns.org/doi/abs/10.3171/2021.3.FOCUS21176

\section{References}

1. Tumialán LM, Pan J, Rodts GE, Mummaneni PV. The safety and efficacy of anterior cervical discectomy and fusion with polyetheretherketone spacer and recombinant human bone morphogenetic protein-2: a review of 200 patients. J Neurosurg Spine. 2008;8(6):529-535.

2. Shields LBE, Raque GH, Glassman SD, et al. Adverse effects associated with high-dose recombinant human bone morphogenetic protein-2 use in anterior cervical spine fusion. Spine (Phila Pa 1976). 2006;31(5):542-547.

3. Baskin DS, Ryan P, Sonntag V, et al. A prospective, randomized, controlled cervical fusion study using recombinant human bone morphogenetic protein-2 with the CORNERSTONE-SR allograft ring and the ATLANTIS anterior cervical plate. Spine (Phila Pa 1976). 2003;28(12):1219-1225.

4. Hofstetter CP, Hofer AS, Levi AD. Exploratory meta-analysis on dose-related efficacy and morbidity of bone morphogenetic protein in spinal arthrodesis surgery. $J$ Neurosurg Spine. 2016;24(3):457-475.

5. Mendenhall SK, Priddy BH, Mobasser JP, Potts EA. Safety and efficacy of low-dose rhBMP-2 use for anterior cervical fusion. Neurosurg Focus. 2021;50(6):E2. 
6. Boden SD, Zdeblick TA, Sandhu HS, Heim SE. The use of rhBMP-2 in interbody fusion cages. Definitive evidence of osteoinduction in humans: a preliminary report. Spine (Phila Pa 1976). 2000;25(3):376-381.

7. Burkus JK, Gornet MF, Dickman CA, Zdeblick TA. Anterior lumbar interbody fusion using rhBMP-2 with tapered interbody cages. J Spinal Disord Tech. 2002;15(5):337-349.

8. de la Garza Ramos R, Nakhla J, Bhashyam N, et al. Trends in the use of bone morphogenetic protein-2 in adult spinal deformity surgery: a 10-year analysis of 54054 patients. Int J Spine Surg. 2018;12(4):453-459.

9. Rocque BG, Kelly MP, Miller JH, et al. Bone morphogenetic protein-associated complications in pediatric spinal fusion in the early postoperative period: an analysis of 4658 patients and review of the literature. J Neurosurg Pediatr. 2014;14(6): 635-643.

10. Saulle D, Fu KM, Shaffrey CI, Smith JS. Multiple-day drainage when using bone morphogenic protein for long-segment thoracolumbar fusions is associated with low rates of wound complications. World Neurosurg. 2013;80(1-2):204-207.

11. Maza NM, Ferrer CE, Qureshi SA, et al. Contained-delivery route and the administration of postoperative steroids following anterior cervical spinal fusion with low-dose rhBMP-2 reduces the magnitude of respiratory compromise. Clin Spine Surg. 2019;32(10):E420-E425.
12. FDA Public Health Notification: Life-Threatening Complications Associated With Recombinant Human Bone Morphogenetic Protein in Cervical Spine Fusion. Food and Drug Administration; 2008.

13. Liu J, Zhang Y, Hai Y, et al. Intravenous and local steroid use in the management of dysphagia after anterior cervical spine surgery: a systematic review of prospective randomized controlled trails (RCTs). Eur Spine J. 2019;28(2):308-316.

\section{Disclosures}

Dr. Shaffrey is a consultant for Medtronic, NuVasive, and SI Bone; has direct stock ownership in NuVasive; holds patents with Medtronic, NuVasive, and Zimmer Biomet; and received clinical or research support from the ISSGF.

\section{Correspondence:}

Christopher I. Shaffrey: christopher.shaffrey@duke.edu.

\section{INCLUDE WHEN CITING}

DOI: 10.3171/2021.3.FOCUS21176. 